\title{
Anterior Inferior Pancreaticoduodenal Artery Running Between the Dorsal and Ventral Pancreas: Morphological and Embryological Viewpoint
}

\author{
Nozomi $\mathrm{Yi}^{1}$, Shuang-Qin $\mathrm{Yi}^{*}{ }^{*}$, , Heng-Xiao Wang ${ }^{1}$, Yuki Ogawa ${ }^{1}$, Tetsuo Ohta ${ }^{3}$ Noriyuki Ozaki $^{2}$ and \\ Masahiro Itoh ${ }^{1}$ \\ ${ }^{I}$ Department of Anatomy, Tokyo Medical University, Tokyo 160-8402, Japan \\ ${ }^{2}$ Departments of Anatomy and Neuroembryology and ${ }^{3}$ Gastroenterological Surgery, University of Kanazawa, Kanazawa \\ 920-8640, Japan
}

\begin{abstract}
Objective: To elucidate the configurational relationship of the anterior inferior pancreaticoduodenal artery (AIPD) and embryological ventral/dorsal pancreas anlagen.

Methods: 64 cadavers were used for morphological observation by gross dissection of the head and uncinate process of the pancreas as well as its vessels. The localization of pancreatic polypeptide-immunoreactive cells was investigated in 22 cadaveric pancreas samples.

Results: In 73.4\% subjects, the AIPD appeared not on the anterior surface of the anterior inferior portion of the pancreatic head, but was covered by pancreatic parenchyme. The islets of Langerhans were irregular in shape, and abundant pancreatic polypeptide-immunoreactive cells were identified in the pancreatic tissues of the posterior region of the AIPD, while in the anterior region of the AIPD, the islets of Langerhans were rounded, and few or no pancreatic polypeptideimmunoreactive cells were identified.

Conclusions: The AIPD may follow the boundary between the dorsal and ventral pancreas, and we can interpret in embryology of the morphological characteristics by which the AIPD is located behind or on the anterior surface of the anterior inferior portion of the pancreatic head.
\end{abstract}

Keywords: Inferior pancreaticoduodenal artery, superior mesenteric artery, ventral pancreatic primordium, uncinate process, pancreatic polypeptide.

\section{INTRODUCTION}

The morphological structure of the pancreas is still an interesting subject for surgeons and anatomists. For surgeons, this is partly because the surgical treatment of pancreatic diseases has generally been considered to be associated with a high incidence of postoperative complications, and has developed to preserve as much of the pancreatic parenchyma as possible. Limited pancreatic resections, such as medial pancreatectomy [1-3], ventral pancreatectomy [4], uncinate process resection [5], superior head resection [6], and inferior head resection [7] have been performed for benign or low-grade malignant neoplasms of the pancreas, such as mucin-producing tumors, and in some cases for pancreatic injury. These procedures allow maximal preservation of normal pancreatic tissue and hence pancreatic endocrine and exocrine functions, and thus result in a better quality of life for patients [8-10]. Therefore surgeons require concrete topographical anatomical knowledge of the head of the pancreas to avoid critical complications; in particular, grasping the morphological

*Address correspondence to this author at the Department of Anatomy and Neuroembryology, University of Kanazawa, Japan; Tel.: +81-76-265-2494; Fax: +81-76-234-4221; E-mail: yixim@med.kanazawa-u.ac.jp structure of the uncinate process and the boundary of the dorsal and ventral pancreas.

The pancreas is composed of dorsal and ventral primordia in embryological view. The ventral primordium, together with the developing bile duct, rotates clockwise behind the duodenum and the dorsal primordium [11]. Consequently, the head of the pancreas can theoretically be divided into two embryological segments: dorsal and ventral primordia. For anatomists, the configurational arrangement and embryological morphology of the pancreas, especially the rotation of the ventral pancreatic primordium and fusion with the dorsal pancreatic primordium, remain uncertain. The vascular anatomy of the pancreaticoduodenal region has been the subject of numerous studies [12, 13]. It has been conjectured that the anterior inferior portion of the pancreatic head may derive from the embryological anlage of the dorsal pancreas [14] and the anterior inferior pancreaticoduodenal artery (AIPD) may follow the boundary between the dorsal and ventral anlage [14-17]; however, this conjecture has not been confirmed in these reports.

In the current study, we focus on elucidating the configurational relationship of the AIPD and the embryological ventral/dorsal pancreas anlagen in autopsy cases, and present a schematic concept of development to interpret the morphogenesis course. 


\section{SUBJECTS AND METHODS}

\section{Cadavers}

As shown in Table 1, the study was divided into three experimental groups. The first research (Research 1) was performed on 64 subjects ( 21 male and 43 female), the ages from 51 to 90 years (median, 85.9 years old), with morphological observation by gross dissection. The data of Research 1 were then employed in the second research (Research 2), and 10 samples from Research 1 were used for an immunohistochemical experiment in Research 2. The third research (Research 3) employed 12 additional subjects (6 male and 4 female) with a mean age of 88.0 years (range, 82-95 years) for immunohistochemistry. respectively. The samples were washed thoroughly for 5 hours under running tap water, and then dehydrated and routinely embedded in paraffin wax. Sections $(4 \mu \mathrm{m})$ were cut and placed on gelatin-coated glass slides.

For Research 3, employing 12 additional subjects, the head of the pancreas with the superior mesenteric artery was removed. The pancreas samples were realigned (in a transverse plane across the AIPD) in the vertical direction of the AIPD and cut into 5-10 major sections. Each section that included the AIPD was cut into 5- to 7-mm-thick slices, washed thoroughly for 5 hours under running tap water, and then processed as in Research 2.

Both Researches 2 and 3 were used to identify pancreatic polypeptide (PP)-, and insulin-immunoreactive cells in

Table 1. Subjects (Cadavers)

\begin{tabular}{|c|c|c|c|}
\hline & Cadavers & Age & Purpose \\
\hline \hline Research 1 & 64 cases $(\mathrm{M}, 21 ; \mathrm{F}, 43)$ & $51 \sim 90$ (mean 85.9y) & Dissecting pancreas \\
\hline Research 2 & 10 cases $(\mathrm{M}, 3 ; \mathrm{F}, 7)$ & $76 \sim 95$ (mean 87.5y) & IHC to detect PP cells \\
\hline Research 3 & 12 cases $(\mathrm{M}, 6 ; \mathrm{F}, 4)$ & $82 \sim 95$ (mean 88.0y) & IHC to detect PP cells \\
\hline
\end{tabular}

All cadavers were selected from bodies used for the research and practice of anatomy at Tokyo Medical University from 2005 to 2007 and were free from diseases of the pancreas and its surrounding areas.

\section{Morphological Observation}

For Research, 1, 64 cadavers were used to dissect the head and uncinate the process of the pancreas as well as its vessels. We focused on the configuration relationship between the arrangement of the anterior inferior border of the pancreatic head and the AIPD. The distances between the AIPD and the anterior inferior border of the head of the pancreas were measured $(\mathrm{mm})$. The distance was $0 \mathrm{~mm}$ when the AIPD was located at the same level at the anterior inferior border of the head of the pancreas, and the distance was positive or negative when the AIPD was located behind or on the anterior surface of the anterior inferior border of the head of the pancreas, respectively (Table 2). pancreas tissues. The origin of the parenchyma was determined to be the embryologically ventral primordium when the islets of Langerhans had irregular contours and contained abundant PP-positive cells. By contrast, it was determined to be the embryologically dorsal primordium when the surrounding islets were round or oval and PPpositive cells were scarce as compared with the opposite segment, or absent [18-20].

\section{Histological and Immunohistochemical Studies}

For the samples in Research 2 and 3, sets of three consecutive sections were stained with hematoxylin and eosin (HE) and immunostained by using antisera to insulin and PP. Immunohistochemical procedures were performed by the avidin-biotin-peroxidase complex (ABC) method according to our previous study [19]. Briefly, the sections were deparaffinized, placed in a glass box filled with $10 \mathrm{mM}$ citrate buffer $(\mathrm{pH} 6.0)$ for $8 \mathrm{~min}$ at $95^{\circ} \mathrm{C}$ by two times

Table 2. Results of Research 1

\begin{tabular}{|c|c|c|c|}
\hline & Cadavers (\%)* & Distance to AIPD** & Average Distance \\
\hline \hline Group 1 & $47 \operatorname{cases}(73.4 \%)$ & $+1 \sim+33 \mathrm{~mm}$ & \\
\cline { 1 - 3 } Group 2 & $2 \operatorname{cases}(3.1 \%)$ & $0 \mathrm{~mm}$ & \multirow{2}{*}{$7.7 \mathrm{~mm}$} \\
\hline Group 3 & $15 \operatorname{cases}(23.4 \%)$ & $-1 \sim-5 \mathrm{~mm}$ & \\
\hline
\end{tabular}

*Total 64 cadavers

**Distance of inferior border of the head of the pancreas to AIPD.

\section{Tissue Specimens}

For Research 2, after the gross dissection of Research 1 was completed, Among 64 subjects, ten were used randomly in an immunohistochemical experiment. Namely, parts of the pancreatic tissues located in anterior and posterior regions of the AIPD, $0.5-1.0 \times 0.5-0.8 \times 0.3-0.5 \mathrm{~cm}^{3}$, were removed, iterative, for antigen retrieval, pretreated in a temperaturecontrolled microwave oven. Endogenous peroxidase activity was inhibited by 30 -min incubation in methanol containing $0.3 \%(\mathrm{v} / \mathrm{v})$ hydrogen peroxide. After rinsing in $0.01 \mathrm{M}$ phosphate-buffered saline (PBS), the sections were blocked with normal goat serum for 1 hour at room temperature (RT), incubated with the primary antibodies overnight at $4{ }^{\circ} \mathrm{C}$ 
in a humidified chamber, and then with secondary antibodies for 1 hour at RT. Subsequently, the avidin-biotin complex (ABComplex/HRP, DAKO, Denmark) was visualized by incubating the sections for $30 \mathrm{~min}$ at $\mathrm{RT}$, after which they were stained for $1 \mathrm{~min}$ with 3-3'-diaminobenzidine and $0.005 \% \mathrm{H}_{2} \mathrm{O}_{2}$, counterstained with Harris' hematoxylin for $50 \mathrm{~s}$, dehydrated in a graded ethanol series and xylene, and cover-slipped with Entellan neu (Merck, Germany).

The primary antibodies were guinea pig anti-human insulin (polyclonal; Dako, USA), and rabbit anti-human pancreatic polypeptide (polyclonal; YLEM, Italy), diluted at 1:200 and ready-to-use, respectively. The secondary antibody was biotinconjugated goat anti- guinea pig IgG (Santa Cruz Biotechnology, Santa Cruz, CA, USA) and biotin-conjugated goat anti-rabbit IgG (Santa Cruz Biotechnology).

The control experiments consisted of the following: (1) omission of primary antiserum, and (2) substitution of primary antibody with $0.05 \mathrm{M}$ Tris-BSA buffer. These controls were carried out at the same time as treatment with the primary antibody.

\section{Ethics}

Written informed consent was obtained from all study subjects. The study protocol conforms to the ethical guidelines of the "World Medical Association Declaration of Helsinki Ethical Principles for Medical Research Involving Human Subjects" adopted by the 18th WMA General Assembly, Helsinki, Finland, June 1964, as revised in Tokyo 2004. The study was approved by the institutional ethical review committee.

\section{RESULTS}

\section{Morphological Observation for Research 1}

Table 2 summarizes the findings of morphological observation. The results were summarized in 3 groups. In group 1, in 47 of 64 cases (73.4\%), the AIPD was not on the anterior surface of the anterior inferior region of the head of the pancreas, but was covered by pancreatic parenchyma, called partial pancreatic tissues in the "pancreatic lip" (Fig. 1). In these 47 cases, the distance between the AIPD and the anterior inferior edge of the head of pancreas was $1 \sim 33 \mathrm{~mm}$. Moreover, in 3 cases, the parenchyma of the pancreatic lip extended to the region of the uncinate process of pancreas. In group 2, in 2 of 64 cases, the AIPD was located at the same level as the anterior inferior border of the pancreatic lip. In group 3, in 15 of 64 cases, the AIPD was lower than the anterior inferior border of the pancreatic lip, and the distance was $1 \sim 5 \mathrm{~mm}$ (Table 2), with the AIPD following the anterior surface of the anterior inferior portion of the pancreatic head.

\section{Histological and Immunohistochemical Research of Research 2 and Research 3}

In Research 2, the islets of Langerhans were irregular in shape, and abundant PP-immunoreactive cells were identified in the pancreatic tissues of the posterior region of the AIPD (Fig. 2). On the other hand, in the anterior region of the AIPD, the islets of Langerhans were rounded, and few or no PP-immunoreactive cells were identified (Fig. 3).

In Research 3, en bloc pancreatic tissues containing the AIPD were used to detect the distribution of PPimmunoreactive cells. It was found that abundant PPpositive cells were distributed in the dorsal region of the AIPD and, in that region, the islets of Langerhans were irregular in shape; however, in the ventral region of the AIPD, there were few or no PP-immunoreactive cells, and the islets of Langerhans were rounded (Fig. 4).

\section{DISCUSSION}

This study demonstrates that AIPD was located in the boundary of the embryological ventral and dorsal pancreatic primordia, and the course of morphogenesis were first interpreted from a developmental view. To understand this, the configuration relationship of the pancreatic primordium with its vessels needs to be elucidated.

First, the origin of the inferior portion of the head of the pancreas should be located from a developmental view. We
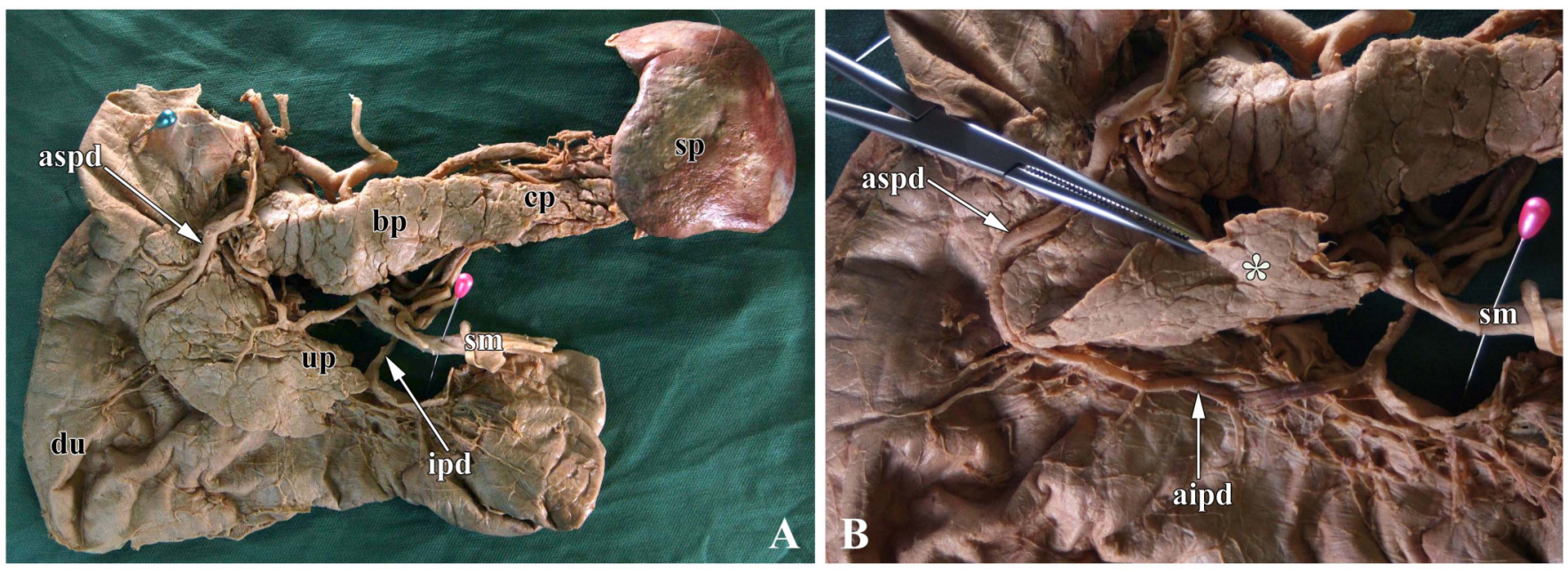

Fig. (1). Photograph of pancreas indicating the anterior inferior pancreaticoduodenal artery (aipd) covered by the lower portion (black *) of the pancreatic head (A), up from the lower portion (white *), appearance of AIPD (B). aspd, anterior superior pancreaticoduodenal artery; bp, body of the pancreas; cp, cauda of the pancreas; du, duodenum; ipd, inferior pancreaticoduodenal artery; sm, superior mesenteric artery; sp, spleen; up, uncinate process. 

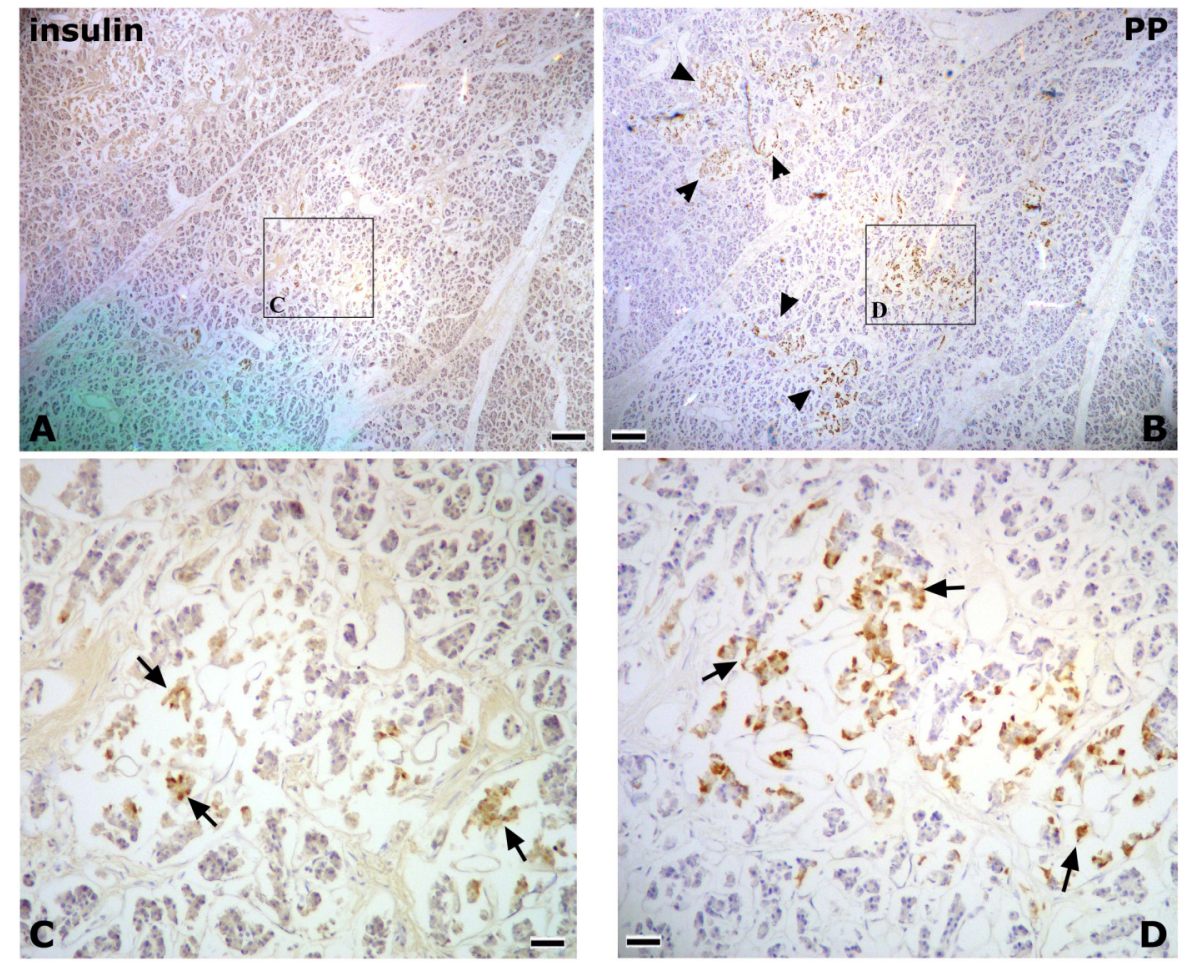

Fig. (2). Immunohistochemical analysis of the distribution of insulin- (A), and pancreatic polypeptide- (PP) (B) immunoreactive cells in the posterior region of AIPD. Arrowheads in $\mathbf{B}$ indicate irregular islets of Langerhans. $\mathbf{C}$ and $\mathbf{D}$ : higher magnification of the area indicated in $\mathbf{A}$ and $\mathbf{B}$, respectively. Arrows: insulin- $(\mathbf{C})$ or PP- (D) immunoreactive cells. Scale bar: $\mathbf{A}$ and $\mathbf{B}=500 \mu \mathrm{m} ; \mathbf{C}$ and $\mathbf{D}=100 \mu \mathrm{m}$.

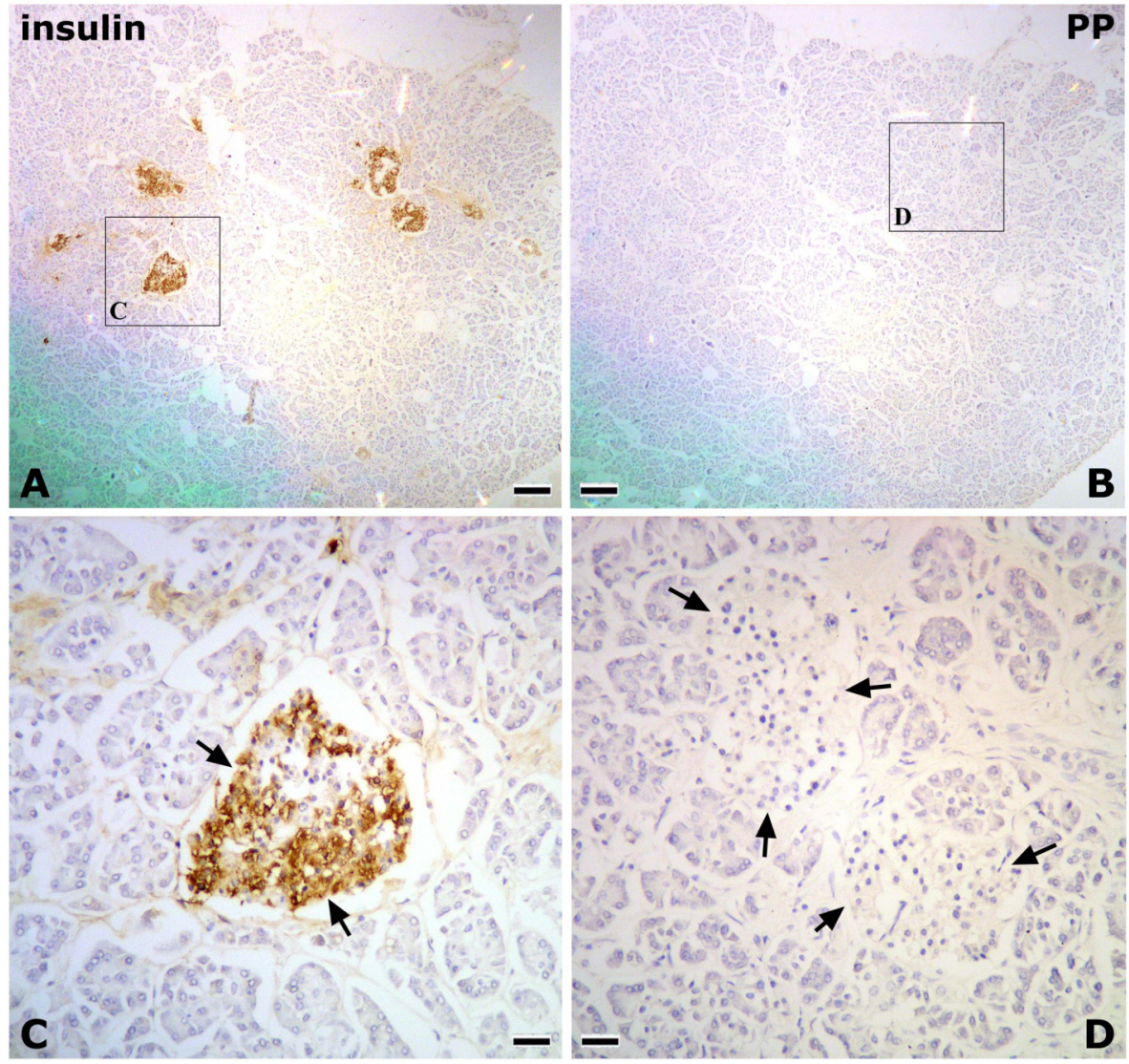

Fig. (3). Immunohistochemical analysis of the distribution of insulin- (A), and pancreatic polypeptide- (PP) (B) immunoreactive cells in the anterior region of AIPD. $\mathbf{C}$ and $\mathbf{D}$ : higher magnification of the area indicated in $\mathbf{A}$ and $\mathbf{B}$, respectively. Arrows: insulin-(C) or PP-(D) immunoreactive cells. Scale bar: $\mathbf{A}$ and $\mathbf{B}=500 \mu \mathrm{m} ; \mathbf{C}$ and $\mathbf{D}=100 \mu \mathrm{m}$. 


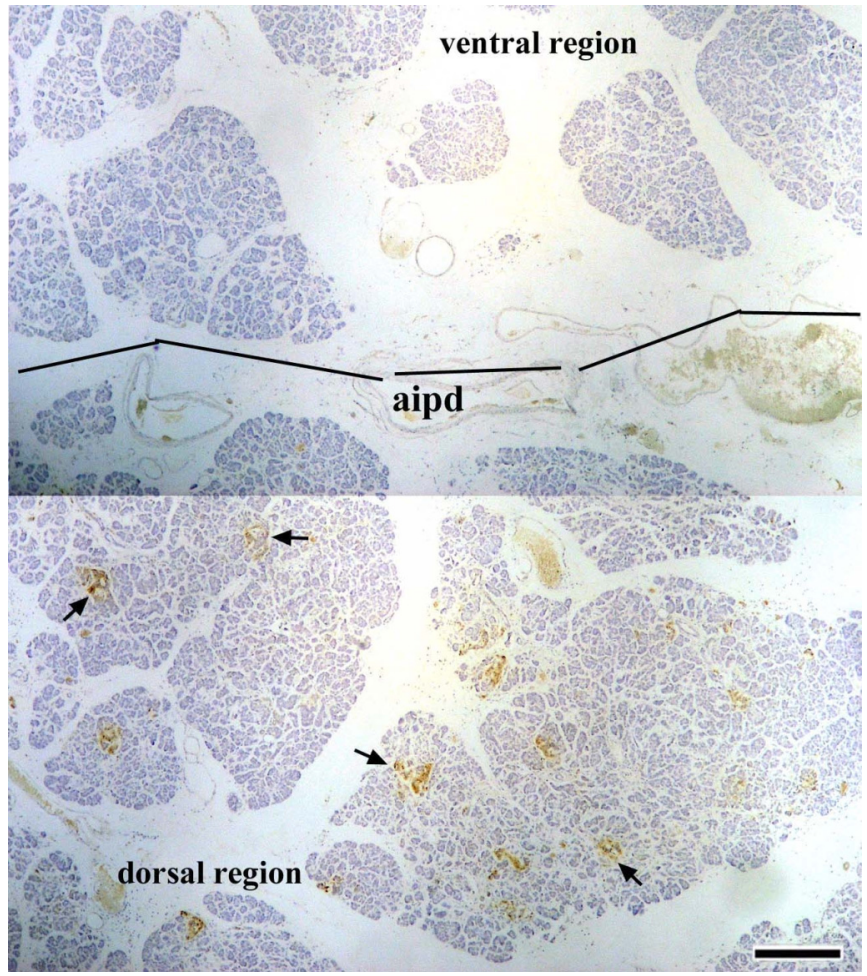

Fig. (4). Immunohistochemical analysis of the distribution of pancreatic polypeptide-immunoreactive cells in dorsal and ventral regions of the anterior inferior pancreaticoduodenal artery (AIPD). PP-immunoreactive cells were only distributed in the dorsal region. Arrows: PP-immunoreactive cells. Scale bar: $200 \mu \mathrm{m}$.

do not support the classical description in which the ventral pancreas constitutes most of the heads of the gland, except for the anterior portion of its superior third, which was derived from the dorsal pancreas [21]. In our study, the anterior inferior portion of the pancreatic head showed fewer or no PP-immunoreactive cells, indicating that it arose from the embryological dorsal primordium, whereas the posterior inferior portion showed PP-rich islets, indicating that it was derived from the ventral primordium, and the boundary in the lower portion of the pancreatic head was the AIPD. Our findings are similar to previous reports by Tadokoro et al. [22] and Uchida et al. [8]; however, the relationship between the AIPD and the dorsal/ventral pancreas primordium were not mentioned in these reports.

Furthermore, the uncinate process of the pancreas is generally described as having an exclusive embryonic origin from the ventral primordium [23, 24], but our findings showed that the uncinate process is of double embryonic origin, the ventral and dorsal pancreas. Uchida et al. [8] also reported that the uncinate process consisted of both the ventral (upper two-thirds) and dorsal primordia in five of eight autopsy specimens. In Fig. (5), during development, the size of the ventral and dorsal primordia influences the inclination of the ventral and dorsal pancreas in the uncinate process. The uncinate process is therefore not derived solely from the primordium, dorsal or ventral.

Second is the course of the AIPD, along the anterior surface or behind the lower portion of the pancreatic head. In current textbooks and atlases of anatomy, it has been described or shown that the AIPD runs along the anterior surface of the lower portion of the pancreatic head $[25,26]$; however, in the present study, the AIPD ran not with the anterior surface, but behind the lower portion of the pancreatic head in $73.4 \%$ of subjects in our investigation. Similar results were reported by Nagai [8] and the lower portion was called the "mentum" or "chin" of the pancreas. Nagai [8] conjectured that the anterior inferior portion of the pancreatic head may derive from the embryological anlage of the dorsal pancreas; however, the conjecture was not confirmed in this report. According to our results, PP-poor islets are distributed in the anterior inferior portion of the pancreatic head; therefore, the "mentum" of the pancreas was proven to derive from the embryological anlage of the dorsal pancreas by immunohistochemistry.

Third, it was a very important discovery that the embryological ventral and dorsal pancreatic anlagen are irrigated by the superior mesenteric artery and celiac trunk, respectively. Experimental animal, house musk shrew (Suncus murinus), which are common insectivores, has shown morphological characteristic very similar to those of humans than other species, i.e., mice, rats, and is useful as a model of human physiology and pathophysiology [20, $27-$ 29]. According to our previous studies, the pancreases do not fuse as in humans or other species, and the two pancreases are located on the right and left of the common bile duct in Suncus murinus. The right and left pancreases are supplied by the superior mesenteric artery and celiac trunk, respectively [27], and the distribution of PP immunoreactive cells is limited to the islets of Langerhans of the right pancreas, but not the left pancreas [20]. It is suggested that the right pancreas originates from the embryological anlage of the ventral pancreas (PP-rich islets); hence, the ventral pancreatic anlage is irrigated by the superior mesenteric artery, and the dorsal anlage is irrigated by the celiac trunk in Suncus murinus. Baetens et al. [18] also reported that, in rats, the pancreatic regions that provided the two different types of islets have been shown to be irrigated by different arterial systems, the celiac artery for the dorsal region and splenic lobes and the superior mesenteric artery for the ventral region and the duodenal lobe, which were demonstrated to have PP-rich islets.

Furthermore, our recent study indicated that carcinomas of the ventral and dorsal pancreas exhibit different patterns of lymphatic spread. Namely, when the tumor is confined to the ventral pancreas domain, lymph node metastases were limited to areas with the superior mesenteric artery besides peripancreatic lymph nodes. When the tumor was in the dorsal pancreas domain, the lymph node metastases were limited to areas along the common hepatic artery and the hepatoduodenal ligament besides peripancreatic lymph nodes [30]; therefore, it is suggested that, during embryonic development, the ventral and dorsal pancreatic anlagen are irrigated by the superior mesenteric artery and celiac trunk, respectively.

Consequently, it is not difficult to understand the mechanism of the AIPD course along the boundary between the dorsal and ventral anlagen, and why it is located behind or on the anterior surface of the lower portion of the pancreatic head. As shown in Fig. (5), during embryonic development, the dorsal and ventral pancreatic primordia arise from ventral and dorsal mesogastrium, irrigated by the 

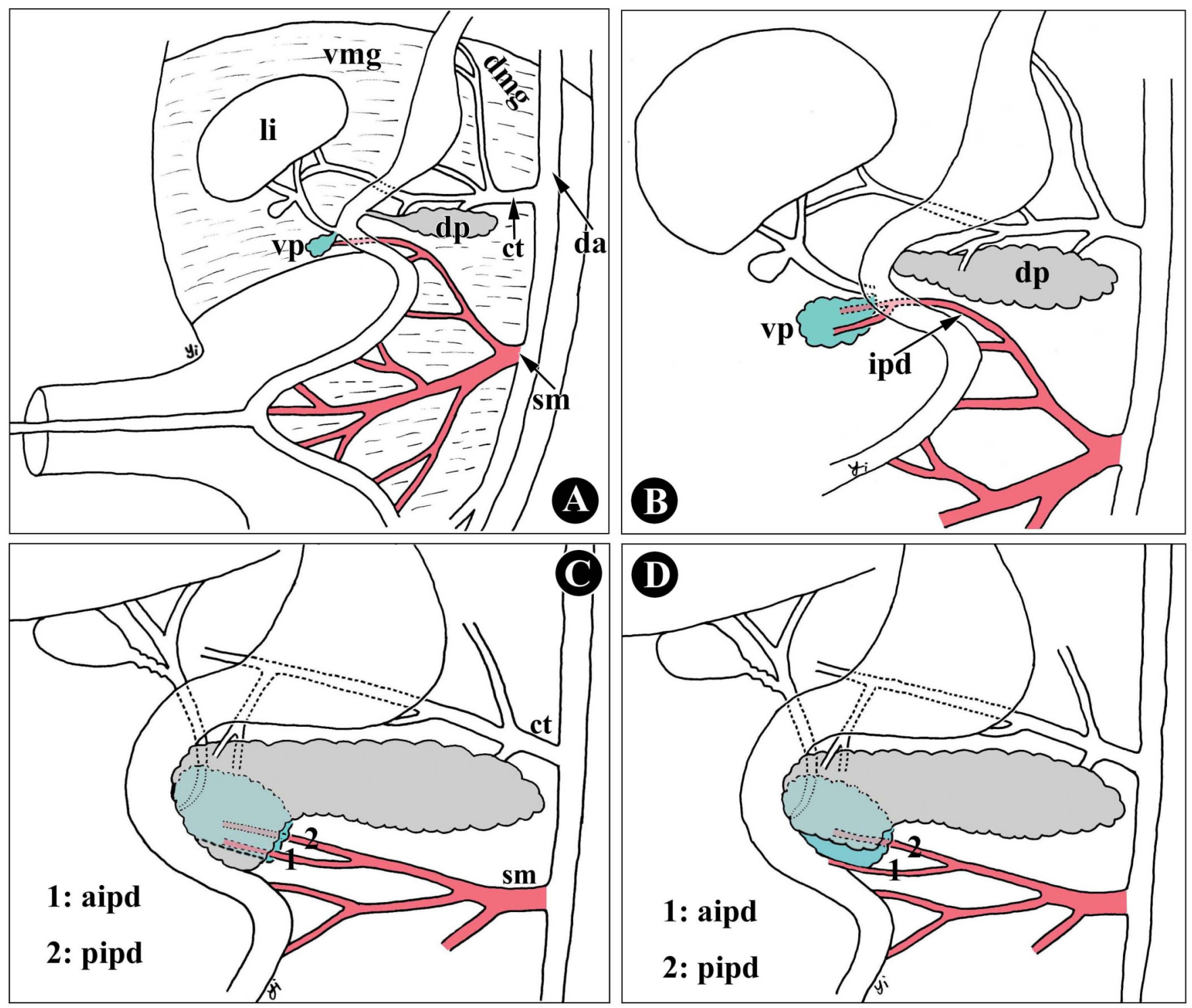

Fig. (5). Schematic representation indicting the morphogenesis course of the arrangement of the AIPD and the ventral and dorsal pancreatic primordia. AIPD/PIPD, anterior/posterior inferior pancraticoduodenal artery; ct, celiac trunk; da, dorsal aorta; dmg/vmg, dorsal/ventral mesogastrium; dp/vp, dorsal/ventral pancreatic bud; ipd, inferior pancreaticoduodenal artery; li, liver; sm, superior mesenteric artery.

superior mesenteric artery and celiac trunk (Fig. 5A, B). In this fusion process of two primordia, the ventral primordium has, together with the developing common bile duct, rotates clockwise behind the duodenum and the dorsal primordium. Thus, the topographic position of the two primordia has inverted in the adult pancreas: the present anterior segment originates from the embryologically dorsal primordium, while the posterior segment from the ventral primordium [31]; however, the overlap pattern of the ventral and dorsal pancreas has not been unified. The factors influencing the patterns include the size of the ventral pancreas, rotating angles and directions on the ventral pancreas, and the craniocaudal distance (with upper and lower) of the ventral and dorsal pancreas or the distance of the orifice of Santorini's duct and Wirsung's duct, that is to say, both the ventral pancreas and its vessels, including AIPD, might be covered by the dorsal pancreas when the ventral pancreas is smaller, or the distance between the orifice of Santorini's duct and
Wirsung's duct is relatively distant, or the rotating direction turns slightly cephalad (Fig. 5C). In contrast, the AIPD appears on the anterior surface of the anterior inferior portion of the pancreatic head (Fig. 5D).

In summary, the current study adds further insight into and knowledge of the embryonic development and anatomy of the ventral and dorsal pancreas. The AIPD may follow the boundary between the dorsal and ventral pancreatic anlagen, and we have interpreted embryologically why the AIPD is located on the anterior surface or behind the anterior inferior portion of the pancreatic head. Our conclusion may be of direct or indirect relevance to the pancreatic surgeon.

\section{ACKNOWLEDGEMENTS}

This study was supported in part by Grant 17590155 from the Ministry of Education, Culture, Sports, Science and Technology of Japan. 


\section{ABBREVIATIONS}

$$
\begin{aligned}
& \text { AIPD }=\text { Anterior inferior pancreaticoduodenal artery } \\
& \text { PBS }=\text { Phosphate-buffered saline } \\
& \text { PP } \\
& \text { RT }
\end{aligned}
$$

\section{REFERENCES}

[1] Fagniez PL, Kracht M, Rotman N. Limited conservative pancreatectomy for benign tumours: a new technical approach. Br J Surg 1988; 75: 719 .

[2] Takada T, Yasuda H, Uchiyama K, Hasegawa H, Iwagaki T, Ymakawa Y. A proposed new pancreatic classification system accoding to segments: operative procedure for a medial pancreatic segmentectomy. J Hepatobiliary Pancreat Surg 1994; 1: 322-5.

[3] Warshaw AL, Rattner DW, Fernández-del Castillo C, Z'graggen K. Middle segment pancreatectomy: a novel technique for conserving pancreatic tissue. Arch Surg 1998; 133: 327-31.

[4] Takada T. Ventral pancreatectomy: Resection of the ventral segment of the pancreas. J Hepatobiliary Pancreat Surg 1993; 1: 36-40.

[5] Watanabe G, Matsuda M, Kajiyama Y et al. Clinical aspects for the resection of the uncinate process of the pancreas. J Bil Panc 1991; 12: 1369-73. (in Japanese)

[6] Kamiya J, Nagino M, Kanai M, et al. Superior segmentectomy of the pancreatic head. Operation 1999; 53: 1367-71. (in Japanese)

[7] Nakagohri T, Asano T, Takayama W, et al. Resection of the inferior head of the pancreas: report of a case. Surg Today 1996; 26: $640-4$

[8] Uchida T, Takada T, Ammori BJ, Suda K, Takahashi T. Threedimensional reconstruction of the ventral and dorsal pancreas: a new insight into anatomy and embryonic development. J Hepatobiliary Pancreat Surg 1999; 6: 176-80.

[9] Kimura W. Surgical anatomy of the pancreas for limited resection. J Hepatobiliary Pancreat Surg 2000; 7: 473-9.

[10] Sakamoto Y, Nagai M, Tanaka N, et al. Anatomical segmentectomy of the head of the pancreas along the embryological fusion plane: a feasible procedure? Surgery 2000; 128: $822-31$

[11] Skandalakis LJ, Rowe JS Jr, Gray SW, Skandalakis JE. Surgical embryology and anatomy of the pancreas. Surg Clin North Am 1993; 73: 661-97.

[12] Bertelli E, Di Gregorio F, Bertelli L, Orazioli D, Bastianini A. The arterial blood supply of the pancreas: a review. IV. The anterior inferior and posterior pancreaticoduodenal aa., and minor sources of blood supply for the head of the pancreas. An anatomical review and radiologic study. Surg Radiol Anat 1997; 19: 203-12.

[13] Murakami G, Hirata K, Takamuro T, Mukaiya M, Hata F, Kitagawa S. Vascular anatomy of the pancreaticoduodenal region: A review. J Hepatobiliary Pancreat Surg 1999; 6: 55-68.
[14] Nagai H. Configurational anatomy of the pancreas: its surgical relevance from ontogenetic and comparative-anatomical viewpoints. J Hepatobiliary Pancreat Surg 2003; 10: 48-56.

[15] Villemin F. Sur la circulation arterielle du duodenum. CR Ass Anat 1921; 16: 223-8.

[16] Woodburne RT, Olsen LL. The arteries of the pancreas. Anat Rec 1951; 111:255-70.

[17] Meyer P. Etude du tronc arteriel pancreatico-duodeno-jejunal. CR Ass Anat 1953; 40: 99-119.

[18] Baetens D, Malaisse-Lagae F, Perrelet A, Orci L. Endocrine pancreas: three-dimensional reconstruction shows two types of islets of Langerhans. Science 1979; 206: 1323-5.

[19] Yi SQ, Tanaka S, Tanaka A, Shimokawa T, Ru F, Nakatani T. An extremely rare inversion of the preduodenal portal vein and common bile duct associated with multiple malformations: Report of an adult cadaver case with a brief review of the literature. Anat Embryol (Berl) 2004; 208: 87-96.

[20] Yi SQ, Akita K, Ohto T, et al. Cellular localization of endocrine cells in the adult pancreas of the house musk shrew, Suncus murinus: a comparative immunohistochemical study. Gen Comp Endocrinol 2004; 136: 162-70.

[21] Russu IG, Vaida A. New findings on the development of the pancreas. Acta Anat (Basel) 1959; 38: 114-25. (in German)

[22] Tadokoro H, Kozu T, Toki F, Kobayashi M, Hayashi N. Embryological fusion between the ducts of the ventral and dorsal primordia of the pancreas occurs in two manners. Pancreas 1997; 14: 407-14.

[23] O'Rahilly R, Müller F. A model of the pancreas to illustrate its development. Acta Anat 1978; 100: 380-5.

[24] Skandalakis JE, Gray SW, Ricketts R, Skandalakis LJ. The pancreas. In: Skandalakis JE, Gray SW, Eds. Enbryology for surgeons: The embryological nasis for the treatment of congenital anomalies. Williams and Wilkins, Maryland, 1994: 366-404.

[25] Moore KL, Dalley AF. Chapter 2: Abdomen. In: Moore KL, Dalley AF, Eds. Clinically Oriented Anatomy, 4th. Tokyo, Lippincott Williams \& Wilkins, 1999: 240-59.

[26] Borley NR. Section 8, Abdomen and Pelvis: Chapter 70, Pancreas. In: Standring S, Ed. Gray's Anatomy. 40th. New York, Churchill Livingstone, 2008: 1183-7.

[27] Yi SQ, Miwa K, Ohta T, et al. Innervation of the human pancreas from the perspective perineural invasion of pancreatic cancer. Pancreas 2003; 27: 225-9.

[28] Yi SQ, Ohta T, Miwa K, et al. Surgical anatomy of the innervation of the major duodenal papilla in humans and Suncus murinus, from the perspective of preserving innervation in organ-saving procedures. Pancreas 2005; 30: 211-7.

[29] Yi SQ, Shimokawa T, Akita K, et al. Anatomical study of the pancreas in the house musk shrew, Suncus murinus, with special reference to the blood supply and innervation. Anat Rec A Discov Mol Cell Evol Biol 2003; 273: 630-5.

[30] Kitagawa H, Ohta T, Makino I, et al. Carcinomas of the ventral and dorsal pancreas exhibit different patterns of lymphatic spread. Front Biosci 2008; 13: 2728-35.

[31] Slack JM. Developmental biology of the pancreas. Development 1995; 121: 1569-80. 